\title{
Utilização de Pistas Proprioceptivas e Movimentos Oculares na Doença de Machado Joseph: Estudo de Caso
}

\author{
Utilization of the Proprioceptive Runway and Ocular Movements In the Machado-Joseph Disease: Case Study
}

\author{
Ariadine Cristine Alves Mendes de Oliveira ${ }^{1}$, Aline Cristina Britto Viana ${ }^{1}$, \\ Rita Helena Duarte Dias Labronici
}

\begin{abstract}
RESUMO
A Doença de Machado-Joseph, também conhecida como ataxia espinocerebelar do tipo 3, é considerada uma doença hereditária, progressiva, categorizada como uma ataxia cerebelar autossômica dominante. Sua sintomatologia está relacionada a um lento progresso, principalmente de manifestações clínicas como: alterações oculomotoras, espasticidade, tremores, marcha atáxica, entre outros. O presente estudo propôs um protocolo fisioterapêutico para melhora do equilíbrio e atividade de vida diária. Foi utilizada a Escala de Equilíbrio de Berg e a Medida de Independência Funcional, os testes foram realizados por um avaliador cego, antes e após a intervençáo. O protocolo proposto foi realizado através de duas pistas proprioceptivas, com diferentes tipos de pisos e obstáculos, utilizando também movimentos oculares. Neste estudo de caso teve a participação de um indivíduo, 27 anos, do sexo masculino, com o diagnóstico clínico de Doença de Machado Joseph. No início apresentava dificuldade de equilíbrio, marcha com apoio de bengala e ajuda de terceiro. Ao término do tratamento, obteve melhora do equilíbrio, da atividade de vida diária e correção da marcha. A realização do protocolo fisioterapêutico, pode ser mais um recurso para minimizar a progressão da doença, melhorando assim seu equilíbrio e sua independência funcional.
\end{abstract}

Unitermos. Equilíbrio, Fisioterapia, Ataxia, Movimentos Oculares.

Citaçáo. Oliveira ACAM, Viana ACB, Labronici RHDD. Utilização de Pistas Proprioceptivas e Movimentos Oculares na Doença de Machado Joseph: estudo de caso.

\begin{abstract}
The Machado-Joseph disease, also known as spinocerebellar ataxia kind 3 , is considered a hereditary disease, progressive, categorized as an autosomal dominant cerebellar ataxia. Your symptomatology is relation a slow progress, mainly of clinic manifestation as: ocular motor alterations, spasticity, tremors, ataxic march, and others. The present study proposes a physiotherapeutical protocol for improvement of balance and daily activities life. Berg Balance Scale and Functional Independence Measure utilized, the test were accomplished by blind evaluation in pre- and post-intervention. The protocol was accomplished through two avenues proprioceptive with different types of floors and obstacles, also using eye movements. In this case study had the participation of an individual, 27 years, old male with a clinical diagnosis of Machado-Joseph disease. At first had difficulty balancing, walking with a case support and help from third. At the end of treatment, had improved balance, activities of daily life and gait correction. The completion of physical therapy protocol may be more a feature to minimize disease progression, thereby improving its balance and its functional independence.
\end{abstract}

Keywords. Balance, Physiotherapy, Ataxia, Eye Movements.

Citation. Oliveira ACAM, Viana ACB, Labronici RHDD. Utilization of the proprioceptive runway and ocular movements in the Machado-Joseph Disease: case study.

\footnotetext{
Trabalho realizado na Policlínica do Centro Universitário Adventista de Sáo Paulo, Sáo Paulo-SP, Brasil.

1.Fisioterapeutas, Graduadas no Centro Universitário Adventista de São Paulo - UNASP/SP, São Paulo-SP, Brasil.

2.Fisioterapeuta, Mestre em Neurociência - UNIFESP, Responsável pela Disciplina de Neurologia - UNASP/SP, São Paulo-SP, Brasil.
}

Endereço para correspondência: Ariadine Cristine AM de Oliveira Rua Moacir Costa Rifano, 06 CEP 05833-270, São Paulo-SP, Brasil.

E-mail: ariadinefisio@yahoo.com.br

Relato de Caso Recebido em: 07/05/10 Aceito em: 18/04/11 Conflito de interesses: não 


\section{INTRODUÇÃO}

O cerebelo apresenta grande importância para o Sistema Nervoso Central (SNC) pelas suas funçóes e inter-relações que desempenha ${ }^{1}$. As principais funções do cerebelo envolvem coordenação da atividade motora, equilíbrio e tônus muscular. Além disso, o cerebelo é vital no controle das atividades musculares rápidas, correção e aprendizado motor. Doenças e distúrbios cerebelares produzem deficiências na velocidade, amplitude e força do movimento. Dessa forma, a perda dessa área do encéfalo pode resultar em incoordenação dessas atividades motoras $^{2}$.

A ataxia é um sinal neurológico caracterizado pela incoordenação dos movimentos voluntários e é a principal manifestação de uma síndrome cerebelar ${ }^{3}$. Aparece no tronco, nas extremidades, na cabeça, boca e língua (fala), multiarticulações e padrões de movimento são mais afetados do que movimentos de uma única articulaçãó ${ }^{4}$.

As enfermidades heredo-degenerativas, entre elas as ataxias cerebelares autossômicas dominantes, também conhecidas como ataxia espinocerebelares (SCA3), correspondem a extenso grupo de doenças com grande heterogeneidade genética, o que dificulta sobremaneira o diagnóstico clínico. A forma mais comum de SCA3, é a doença de Machado-Joseph ${ }^{5,6}$.

A Doença de Machado-Joseph (DMJ) é considerada uma doença hereditária, progressiva, categorizada como uma ataxia cerebelar autossômica dominante $(\mathrm{ACAD})^{6,7}$. Está ligada a expansão de um trinucleotídio CAG na região codificadora do gene da doença, acarretando a expressão de uma proteína enriquecida em glutamina. Trata-se de uma mutação instável e a correlação entre o número de repetiçóes do códon e a idade de início, com tendência ao fenômeno de antecipação em geraçôes sucessivas $^{8}$.

O primeiro aspecto a ser considerado na abordagem de um paciente com ataxia é a sua forma de início. Quadros agudos tomam obrigatória a exclusão de enfermidades focais do cerebelo, transtornos metabólicos e infecciosos ${ }^{3}$.

Geralmente a sintomatologia da DMJ esta relacionada a um lento progresso, principalmente de manifestaçóes cerebelares acompanhadas de vários outros sintomas como: disartria, alteraçôes oculomotoras, espasticidade, tremores, marcha atáxica, ataxia e disfagia, distonia, fasciculaçóes de língua e face, entre outros?

Pensando em todas as dificuldades que esta DMJ apresenta, foi elaborado um protocolo fisioterapêutico ainda não existente na literatura, que utiliza-se de duas pistas proprioceptivas com e sem barra, utilizando diferentes tipos de pisos e movimentos oculares, com o intuito de melhorar o quadro clínico desta doença.

O objetivo deste estudo foi propor e verificar a influência do protocolo fisioterapêutico no equilíbrio, atividade motora e de vida diária.

\section{MÉTODO}

\section{Estudo de caso}

O presente trabalho trata-se de um estudo de caso, numa pesquisa longitudinal em que o paciente do sexo masculino, de 27 anos, começou a sentir dores constantes nos membros inferiores em fevereiro de 2009, procurou tratamento médico no Hospital São Paulo, no qual foram feitos uma série de exames que obteve o diagnóstico de Ataxia Hereditária Dominante, e passou a fazer tratamento medicamentoso. Em julho de 2009 foi feito o exame genético, resultando no diagnóstico de Ataxia Espinocerebelar tipo 3 (SCA3) Doença de MachadoJoseph (DMJ). Após um curto período de tempo passou a apresentar um quadro clínico de perda de equilíbrio e dificuldade na marcha, passando a utilizar uma bengala e auxílio de terceiros (outra pessoa) para se locomover. Este estudo obteve aprovação do Comitê de ética em Pesquisa sob o parecer no 072/2009 do Centro Universitário Adventista de São Paulo (UNASP-SP). O indivíduo foi avaliado e tratado na Policlínica Universitária do UNASP, onde assinou o Termo de Consentimento Livre e Esclarecido de acordo com a resolução 196/96 do Conselho Nacional de Saúde.

\section{Procedimentos}

Foi proposto um protocolo de tratamento fisioterapêutico aplicado duas vezes por semana, durante seis semanas com duração de 30 minutos por sessão. No qual inicialmente e ao término do estudo houve uma avaliação cega, através da Escala de Equilíbrio Funcional de Berg e a Medida de Independência Funcional (MIF).

A escala de equilíbrio funcional de Berg avalia o 
equilíbrio estático e dinâmico, baseada em 14 itens comuns da vida diária ${ }^{10}$, em que o número 4 é usado para indicar que o paciente realiza a tarefa de forma independente e de acordo com os critérios de tempo e distância, e o número 0 é usado para indicar incapacidade de realizar a tarefa; nesta avaliação é possível o máximo de 56 pontos ${ }^{11}$. Além da Escala de Equilíbrio Funcional de Berg os instrumentos utilizados foram: fita métrica, cronômetro, banco medindo $52 \mathrm{~cm}$ de altura e $62 \mathrm{~cm}$ de comprimento e uma cadeira sem apoio de braços.

A MIF verifica o desempenho do indivíduo para realização de um conjunto de 18 tarefas, referentes às subescalas autocuidados, controle esfincteriano, transferência, locomoção, comunicação e cogniçãao social ${ }^{12} \mathrm{em}$ uma escala de um a sete. Nesta escala são avaliados 18 itens e a pontuação do paciente vai de no mínimo 18 a no máximo 126 pontos. A MIF é dividida em dois escores que se subdividem em itens: 1) escore motor: comer, aprontar-se, banho, vestir parte superior do corpo, vestir parte inferior do corpo, vaso sanitário, controle de bexiga, controle de intestino, transferência da cama para a cadeira de rodas, transferência da cadeira de rodas para o vaso sanitário, transferência no banheiro e chuveiro, marcha/ cadeira de rodas, escadas; 2) escore cognitivo: compreensão, expressão, integração social, resolução de problemas e memória ${ }^{12}$.

O protocolo proposto utiliza-se de duas pistas proprioceptiva com e sem barra. Com uma barra (medindo $26,18 \mathrm{~m}$ de comprimento e $1,19 \mathrm{~cm}$ de largura, sendo que entre um obstáculo e outro a pista media 4,02 m de comprimento): 2 pisos de areia, 1 piso de pedra, 2 pisos de terra e 1 degrau de $15 \mathrm{~cm}$ de altura. Sem barra (corredor interno da policlínica com piso, medindo 18 m de comprimento) com os obstáculos no chão: bastôes, colchonetes, degrau $22 \mathrm{~cm}$ de altura e $44 \mathrm{~cm}$ de largura e cama elástica. Junto com o treino de marcha na pista o paciente foi orientado través de comandos verbais e visuais com a utilização de bola colorida e a própria voz da fisioterapeuta ao olhar para frente, para trás, ora para direita, ora para esquerda e ponto fixo (Figura 1 e 2).

O protocolo consta de seis etapas descritas a seguir:

Etapa 1 - Adaptação nas pistas: paciente foi orientado inicialmente a caminhar sobre a pista proprioceptiva, no início utilizou sua bengala só para adaptação.

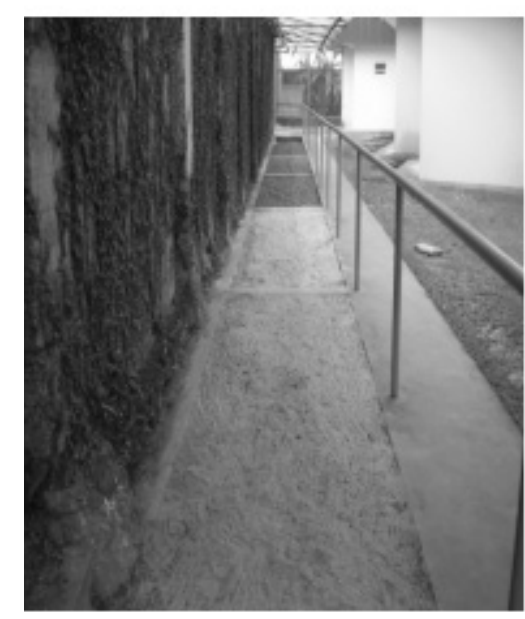

Figura 1. Pista Proprioceptiva com barra.

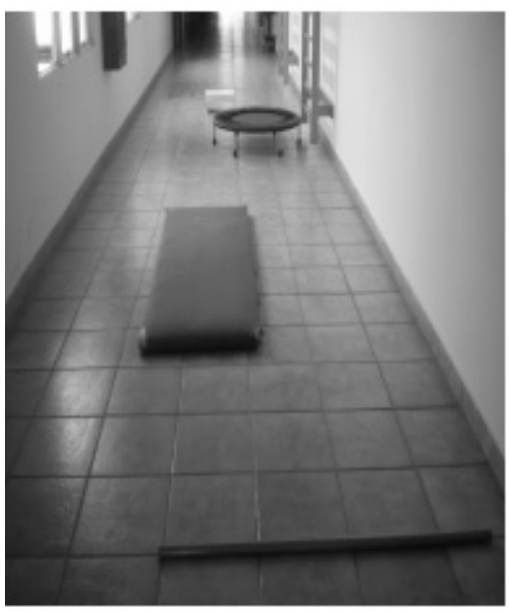

Figura 2. Pista Proprioceptiva sem barra.

Etapa 2 - Correção da marcha: o paciente recebia orientações da fisioterapeuta para dissociação de cinturas, apoio de pés, atenção para os movimentos e não olhar para o chão.

Etapa 3 - Movimentos oculares: foi proposto ao paciente para que durante o treino de marcha o mesmo realizasse movimentos oculares através de estímulos verbais da própria fisioterapeuta, no qual tinha que olhar ora para direita, ora para esquerda e ponto fixo.

Etapa 4 - Movimentos de cabeça: assim como na etapa anterior em que o paciente realizava movimentos oculares durante a marcha, o mesmo passou a realizar também movimentos de cabeça, com o olhar para frente, para trás, para direita e para esquerda.

Etapa 5 - Movimentos oculares e de cabeça com utilização de bola colorida e comando de voz: de acordo com a evoluçáo do paciente, foi pedido ao mesmo para que durante o treino de marcha acompanhasse os movimentos 
realizados pela fisioterapeuta com bola (direita, esquerda, para cima e para baixo).

Etapa 6 - Circuito: foi elaborado no corredor interno da Policlínica em uma distância de 18 metros, um circuito constando quatro tipos de obstáculos, que de forma progressiva foi aumentando o número de obstáculos que eram dispostos aleatoriamente. Inicialmente foram utilizados os bastóes, e o paciente tinha que passar sobre e entre eles colocados na pista. Depois com a sua evolução foram acrescentados os colchonetes, e o mesmo tinha que passar sobre eles além dos bastóes. Posteriormente foi acrescentado um degrau de $22 \mathrm{~cm}$ de altura e $44 \mathrm{~cm}$ de largura, no qual o paciente tinha que subir e descer alternando os pés de apoio no degrau ao longo do trajeto estipulado. E por fim foi colocada em frente ao espaldar (para apoio e segurança) uma cama elástica, sendo que o paciente tinha que movimentar-se para os lados, para frente e trás fazendo descarga de peso.

Durante todas as sessóes foram realizados $15 \mathrm{mi}$ nutos de tratamento na pista proprioceptiva com barra e 15 minutos na pista proprioceptiva sem barra. Cada uma das sessôes obedeceu ao nível de adaptação do paciente nas pistas. A aferição da pressão arterial era realizada antes e após as sessóes.

\section{RESULTADOS}

Os resultados da presente pesquisa basearam-se na avaliação antes e após a aplicação do protocolo de tratamento, no qual foi observado uma melhora no final da intervenção através da Escala de Berg, que apresentava inicialmente uma pontuação de 43 , e ao término do tratamento obteve a pontuação máxima sendo de 56 pontos. Na MIF o paciente obteve inicialmente resultado de 114 pontos e após a intervenção obteve 122 pontos.

No treino de marcha na pista proprioceptiva o paciente fez uso de bengala apenas no primeiro dia, pois sentia-se mais seguro nas demais sessóes. $\mathrm{O}$ mesmo realizava a caminhada com disbasia e ausência de dissociação de cinturas, e nas três últimas sessóes já apresentava diminuição da disbasia e melhora na dissociação de cinturas (que foram avaliados e corrigidos de forma observacional, através de filmagens em todas as sessôes.

No degrau o paciente também apresentou certa dificuldade para subir e descer com associação de movi- mentos oculares e de cabeça. E nas últimas sessóes apresentava menos dificuldade.

Sendo assim houve melhora do equilíbrio e da independência funcional em um período de seis semanas.

\section{DISCUSSÃO}

O programa de exercícios, utilizando os movimentos oculares propostos, sugere ser uma estratégia simples e estimuladora, podendo estimular todas as partes do cerebelo, assim como algumas mais especificamente, podendo reintegrar e funcionalizar áreas do tronco cerebral e cerebelo responsáveis pela ataxia cerebelar dos indivíduos portadores de DMJ. Poderá apresentar resultados significativos, estando adequadamente fundamentada nos mais recentes modelos teóricos neurofuncionais ${ }^{6}$.

O tratamento foi baseado em um protocolo tendo como base duas pistas proprioceptivas e movimentos oculares para o tratamento de ataxia espinocerebelar na DMJ. O desenvolvimento do protocolo foi realizado em seis etapas, duas vezes por semana durante seis semanas com duração de 30 minutos, obtendo resultados positivos, pois a doença encontrava-se no estágio inicial.

A proposta de tratamento para ataxia cerebelar na marcha de indivíduos portadores de DMJ, utilizando os movimentos oculares, se fundamenta em algumas possibilidades e segue um modelo estruturado em etapas para a resolução da problemática: 1 - existência de programas de reabilitação funcional por meio de exercícios/atividades específicas; 2 - substrato teórico para fundamentação da proposta; 3 - confirmação da hipótese teórica por estudos específicos; 4 - integração neuroanatômica e neurofuncional entre os movimentos oculares e áreas relacionadas à marcha no SNC; 5 - recuperação funcional de áreas prejudicadas do SNC pela utilização de movimentos oculares ${ }^{6}$.

Um programa de tratamento fisioterapêutico pode ser útil para ajudar a manter a funçáo do portador de doença cerebelar degenerativa com intuito de alcançar grau de independência, cujo nível reside nos diferentes fatores demonstrados nos casos em que há uma determinação progressiva $^{13,14}$. No presente estudo os resultados obtidos foram positivos para independência do paciente, pois, no inicio ele só andava com auxílio de bengala e ajuda de terceiros, e após o tratamento encontrava-se independente. 
Isto pôde ser observado através dos resultados da MIF.

Foi realizado um estudo com indivíduos com degeneração cerebelar primária autossômica dominante que submeteram-se a um protocolo experimental que constava em caminhar sobre um circuito de 18 pedras irregularmente dispostas. Os indivíduos deveriam efetuar as passadas, fazendo um ensaio de movimentos sacádicos ao longo das pedras antes de iniciar uma caminhada. Os movimentos oculares e as passadas foram monitorados ${ }^{15}$. No protocolo foi utilizado além do piso de pedras, piso com areia, terra e degrau. No qual, foi observado que estas diferenças de piso resultaram em uma melhora do equilíbrio e da autoconfiança para o paciente andar sem auxílio de bengala, ajuda de terceiros e também ter mais controle postural.

Os autores dizem que no controle postural existe também a parcela de contribuição do cerebelo, impulsos originados em receptores das articulações, tendôes, músculos, pele e também de órgãos terminais do sistema visual, auditivo e vestibular interagindo com o cerebelo para que ocorra a influência do mesmo sobre a atividade muscular concretizando sua importância no controle do movimento ${ }^{16,17}$.

No processo de integração sensorial, as informações sensoriais são integradas pelo sistema de controle postural e pesos (valor de importância) são dados a cada uma destas informaçóes visual, vestibular e somatossensorial para tornar o controle postural um processo mais flexível, em função das constantes mudanças na relação do indivíduo com o ambiente ${ }^{18}$. Estes pacientes podem ter dificuldades sensoriais se não utilizarem atividades que estimulem principalmente o equilíbrio, postura e movimentos oculares. Pensando nisso foi utilizado comando verbal, visual e alterações de percepçáo quanto ao desnível de pisos e ambiente no protocolo proposto.

No decorrer dos atendimentos o paciente teve início a um quadro depressivo, e por conta deste fator, foi aplicado um método de relaxamento, em um ambiente fechado, calmo, com iluminação baixa, onde o mesmo mantinha os olhos fechados, prestando atenção apenas na voz da fisioterapeuta, que pedia-o para se concentrar e imaginar que estava em uma ambiente de seu agrado realizando movimentos sem qualquer dificuldade, e ao final pedia the para realizar movimentos articulares len- tamente. Este método de relaxamento foi essencial para a continuidade do protocolo, sendo assim não havendo alteraçóes nos resultados obtidos durante o período estipulado do tratamento.

O relaxamento ativa o cerebelo e as vias cerebelares, sendo um princípio fundamental em um programa de exercícios físicos ${ }^{6}$. Por tanto, sugerimos para os próximos estudos que utilizem também o relaxamento durante a intervenção com pacientes de ataxia espinocerebelar na DMJ.

No estudo os resultados obtidos foram em apenas seis semanas, possibilitando ter uma melhora no equilíbrio do paciente, que foi analisado através da Escala de Berg, no qual foi observado que para um jovem que estava dependente da bengala e da ajuda de terceiros favoreceu muito para sua locomoção. Entretanto, sugerimos novos estudos que investiguem a efetividade do protocolo e que utilizem uma demanda maior de pacientes com diagnóstico de DMJ para obtenção de maiores resultados.

\section{CONCLUSÃO}

A proposta do protocolo fisioterapêutico com as utilizaçôes das pistas proprioceptivas e movimentos oculares, foram de fundamental importância para a melhora do equilíbrio e das atividades de vida diária do paciente estudado com a DMJ.

\section{REFERÊNCIAS}

1.Bower JM, Parsons LM. O Cerebelo: reconsiderado. Sci Am Bras 2003;16:66-73.

2.Lundy-Ekman L. Neurociência: fundamentos para a reabilitação. 2a . ed. Rio de Janeiro: Guanabara Koogan, 2004, 447p.

3. Godeiro Junior CO, Felicio AC, Silva SMA, Borges V, Ferraz HB. Abordagem clínica de pacientes com ataxia. Rev Neurocienc. 2007;15:70-5.

4.Umphred DA. Reabilitação Neurológica. 4a Ed. Barueri, São Paulo: Manole, 2004, 765p.

5.Teive HAG, Arruda WO. A família Drew de Walworth: um século após a avaliação inicial finalmente o diagnóstico Doença de Machado-Joseph. Arq Neuropsiquiatr 2004;62:177-80.

http://dx.doi.org/10.1590/S0004-282X2004000100034

6. Rotnes WGV, Orsini M, Bastos VH, Freitas MRG, Nascimento O, Mello MP, et al. Utilização dos movimentos oculares no tratamento da ataxia cerebelar na doença de Machado-Joseph. Rev Neurocienc 2008;16:53-61.

7.Mumenthaler M, Mattle H, Toub E. Neurologia. 4a ed. Rio de Janeiro: Guanabara Koogan, 2007, 299p. 
8.Cambier J, Masson M, Deher H. Neurologia. 11 ${ }^{\mathrm{a}}$ ed. Rio de Janeiro: Guanabara Koogan, 2005, 192p.

9.Busanello AR, Castro SAFN, Rosa AAA. Disartria e doença de MachadoJoseph: relato de caso. Rev Soc Bras Fonoaudiol, 2007;12:247-51.

http://dx.doi.org/10.1590/S1516-80342007000300013

10.Miyamoto ST, Lomberdi Junior I, Berg KO, Ramos LR, Natour J. Brazilian version of the Berg balance scale. Braz J Med Biol Res 2004;37:1411-21. http://dx.doi.org/10.1590/S0100-879X2004000900017

11.Leonardi MM, Lopes GJ, Bezerra PP, Borges APO. Impacto do desequilíbrio estático e dinâmico no risco de quedas em indivíduos com ataxia espinocerebelar. Rev Neurocienc 2009;17:178-82.

12.Riberto M, Miyazakj MH, Jorge Filho D, Sakamoto H, Battistella LR. Reprodutibilidade da versão brasileira da medida de independência funcional. Rev Acta Fisiat 2001;8:45-52.

13.Urbscheit, NL. Disfunção cerebelar. In: Umphred DA. Fisioterapia Neuro- lógica. 2 ed. São Paulo: Manole, 1994, p.593-614.

14.Thomson A, Skinner A, Piercy J. Fisioterapia de Tidy. 12. ed. São Paulo: Santos, 1994, p.441-2.

15.Crowdy KA, Kaur-Mann D, Cooper HL, Mansfield AG, Offord JL, Marple-Horvat DE. Rehearsal by eye movement improves visuomotor performance in cerebellar patients. Exp Brain Res 2002;146:244-7.

http://dx.doi.org/10.1007/s00221-002-1171-0

16. Horak FB, Macpherson JM. Postural Orientation and equilibrium. In: Rowell LB, Sherpherd JT. Handbook of physiology. New York: Oxford University Press, 1996, p.255-92.

17.Bankoff ADP, Campelo TS, Ciol P, Zamai CA. Postura e equilíbrio corporal: um estudo das relaçôes existentes. Rev Mov Percep 2006;6:55-70.

18. Oie KS, Kiemel T, Jeka JJ. Multisensory fusion: simultaneous re-weighting of vision and touch for control of human posture. Cog Brain Res 2002;14:164-76. http://dx.doi.org/10.1016/S0926-6410(02)00071-X 